\title{
Associations of Body Composition Measures and Endogenous Sex-Steroid Hormones among Postmenopausal Women
}

\author{
Lene Mellemkjaer ${ }^{*}, 1$, Jane Christensen ${ }^{1}$, Kirsten Frederiksen ${ }^{1}$, Anja Olsen ${ }^{1}$, Paul Bennett ${ }^{2}$, \\ Kim Overvad $^{3}$ and Anne Tjønneland ${ }^{1}$ \\ ${ }^{1}$ Institute of Cancer Epidemiology, Danish Cancer Society, Copenhagen, Denmark \\ ${ }^{2}$ Statens Seruminstitut, Department of Clinical Biochemistry, Copenhagen, Denmark \\ ${ }^{3}$ Department of Clinical Epidemiology, Aarhus University Hospital, Aalborg, Denmark and Department of Cardiology, \\ Aalborg Hospital, Aarhus University Hospital, Aalborg, Denmark
}

\begin{abstract}
Several studies have shown a positive association between body mass index (BMI) and endogenous levels of female sex-steroid hormones, but relationship with the body fat mass and the fat free mass components of BMI has rarely been investigated. In this cross-sectional study, we included 265 women from the Danish "Diet, Cancer and Health" cohort who were postmenopausal and never users of hormone replacement therapy and who previously were included in a nested case-control study on breast cancer. Multiple log-linear regression analyses of the association between anthropometric measurements and serum levels of estrone, estrone sulfate and sex hormone binding globulin (SHBG) were performed. We found positive associations between all the anthropometric measurements included and estrone and estrone sulfate, whereas negative associations were found for SHBG. We confirmed previous findings of associations between BMI and serum levels of estrogen and SHBG among postmenopausal women. Associations were in the same direction for the two components of BMI, body fat mass index (BFMI) and fat free mass index (FFMI), and use of these more detailed measures did not give a better prediction of the hormone levels than use of BMI alone.
\end{abstract}

Keywords: Body fat mass, fat free mass, anthropometric measures, estrogens, SHBG.

\section{INTRODUCTION}

Overweight has consistently been associated with increased risk of postmenopausal breast cancer with most studies having used body mass index (BMI) as a measure of overweight [1]. After menopause plasma estrogens are mainly produced in fat tissue by aromatization of androgens to estrogen, and a positive correlation between BMI and level of plasma estrogens has been found in a number of studies [2-7]. In one of these studies, correlations with fat mass and fat free mass were also investigated and both were found to be positively correlated with estrogen concentrations [7]. The binding protein, sex hormone binding globulin (SHBG), has been found to be negatively correlated with BMI [2-4,6,7] fat mass and fat free mass [7]. The positive association between adiposity and breast cancer may at least partially be explained by increases in endogenous estrogens and decreases in SHBG $[8,9]$.

The fat and the lean component of BMI may be assessed separately by dividing BMI into body fat mass index (BFMI) and fat free mass index (FFMI). Here we have explored the associations between anthropometric measurements including BMI, BFMI, FFMI, body fat percentage (BF\%) as well as waist and hip circumference with female hormone levels

*Address correspondence to this author at the Institute of Cancer Epidemiology, Danish Cancer Society, Strandboulevarden 49, DK-2100 Copenhagen Ø, Denmark; Tel: +45 352576 12; Fax: +45 352577 31; E-mail: lene@cancer.dk among postmenopausal women who previously were included in a breast cancer case-control study nested within the Danish prospective study "Diet, Cancer and Health".

\section{MATERIALS AND METHODS}

\section{Study Population}

This cross-sectional study included postmenopausal women from the "Diet, Cancer and Health" cohort. This prospective cohort consists of 29,875 women born in Denmark and residing in the greater Copenhagen and Aarhus areas who were recruited between December 1993 and May 1997 when they were aged 50-64 years [10]. At baseline, the women attended a study clinic where blood samples and anthropometric measurements were collected and the participants completed a questionnaire on reproductive factors (e.g. number of births, age at birth of first child), health-related issues (e.g. benign breast tumor, use of hormone replacement therapy), social factors (e.g. years of schooling) and lifestyle factors (e.g. smoking habits, alcohol intake). The study protocol was approved by the regional scientific ethics committees on human studies.

Subjects included in the present study were from a casecontrol study on postmenopausal breast cancer nested within the "Diet, Cancer and Health" cohort [11]. Case subjects were identified in the Danish Cancer Registry with a diagnosis of first primary breast cancer between date of visit to the clinic and 31 December 2000. One control was selected 
from the cohort for each case. Control subjects were cancer free at the exact age at breast cancer diagnosis of the case and matched with the cases on age at entry into the cohort (6 months intervals), certainty of postmenopausal status (known or probable) at baseline and use of hormone replacement therapy at baseline. Of 869 women included in the original case-control study, 576 were excluded because they had ever used hormone replacement therapy.

Trained laboratory technicians obtained measurements of height, weight, waist and hip circumference at the study clinics as described previously [12]. In addition, they measured electrical impedance with a BIA 101-F device (Akern/RJL, Florence, Italy), single frequency $(50 \mathrm{~Hz})$. An equation derived from a Danish population living in the same area as the "Diet, Cancer and Health" population was used to calculate BFM and FFM from the impedance measurement [13]. Body fat mass index (BFMI) and fat free mass index (FFMI) was calculated by dividing BFM (kg) and FFM (kg), respectively, by height squared $\left(\mathrm{m}^{2}\right)$, as suggested by Van Itallie [14]. Body fat percent $(\mathrm{BF} \%)$ was calculated as BFM (kg) divided by total weight (kg) and multiplied by 100 .

\section{Laboratory Assays}

From the blood sample, serum was aliquoted into 1-ml tubes stored in the vapor phase in liquid nitrogen containers $\left(-150^{\circ} \mathrm{C}\right)$. Estrone and estrone sulfate was measured in serum by radioimmunoassay $[15,16]$. SHBG was measured in serum by immunofluorometric analysis [17]. Intra- and interbatch coefficients of variation were: $6.9 \%$ and $12.1 \%$, respectively for estrone, $6.6 \%$ and $10.4 \%$ for estrone sulfate and $4 \%$ and $8 \%$ for SHBG.

\section{Statistical Analyses}

All values of hormone concentrations were log-transformed to correct for skewed distribution. Geometric means for hormone values were higher among women who developed breast cancer during follow-up than among women who did not, while SHBG values were lower among those who developed breast cancer. We calculated geometric means of hormone levels within tertiles of each of the anthropometric measurements (BF\%, BFMI, FFMI, BMI, waist and hip circumference). There was a clear decrease in SHBG levels by increasing tertiles of all anthropometric measurements among both controls and cases. The picture was less clear for the estrogen measurements among both controls and cases, but most of the measurements increased from the lowest tertile to the highest tertile of the anthropometric measurements (data not shown). The overall tendencies described above were similar in cases and controls (tests for similar effects $\mathrm{p} \geq 0.15$ ) in accordance with our expectations, and cases and controls were collapsed into one study group.

We performed multiple log-linear regression analyses for the association between anthropometric measurements as the independent variables and log hormone level as the dependent variable with adjustment for potential confounders. Age (linear), smoking (never, past and current) and alcohol intake (per $10 \mathrm{~g}$ ) were considered as confounders. In the initial analyses, each of the anthropometric variables was modelled as a linear spline with knots placed at the quartiles $\left(25^{\text {th }}, 50^{\text {th }}\right.$ and $75^{\text {th }}$ percentiles) and it was tested if the linear splines of the variables could be simplified to straight lines. None of the anthropometric variables showed significant deviations from linearity (all $\mathrm{p}>0.12$ ). The multivariable regression analyses were stratified by time since menopause $(0-5$ years and $6+$ years). Furthermore, we tested if splitting BMI into BFMI and FFMI were more predictive of hormone levels than BMI alone. The statistical analyses were carried out using SAS (SAS Institute, Cary, NC, USA).

Of the 293 subjects, we excluded 11 (4\%), because they had high levels of female hormones, and were likely not to be postmenopausal. In addition, we excluded $9(3 \%)$ with

Table 1. Medians, Geometric Means and 5-95 Percentiles for Age, Anthropometric Measurements and Levels of Estrogens and SHBG among 265 Postmenopausal Women from the Danish "Diet, Cancer and Health" Study

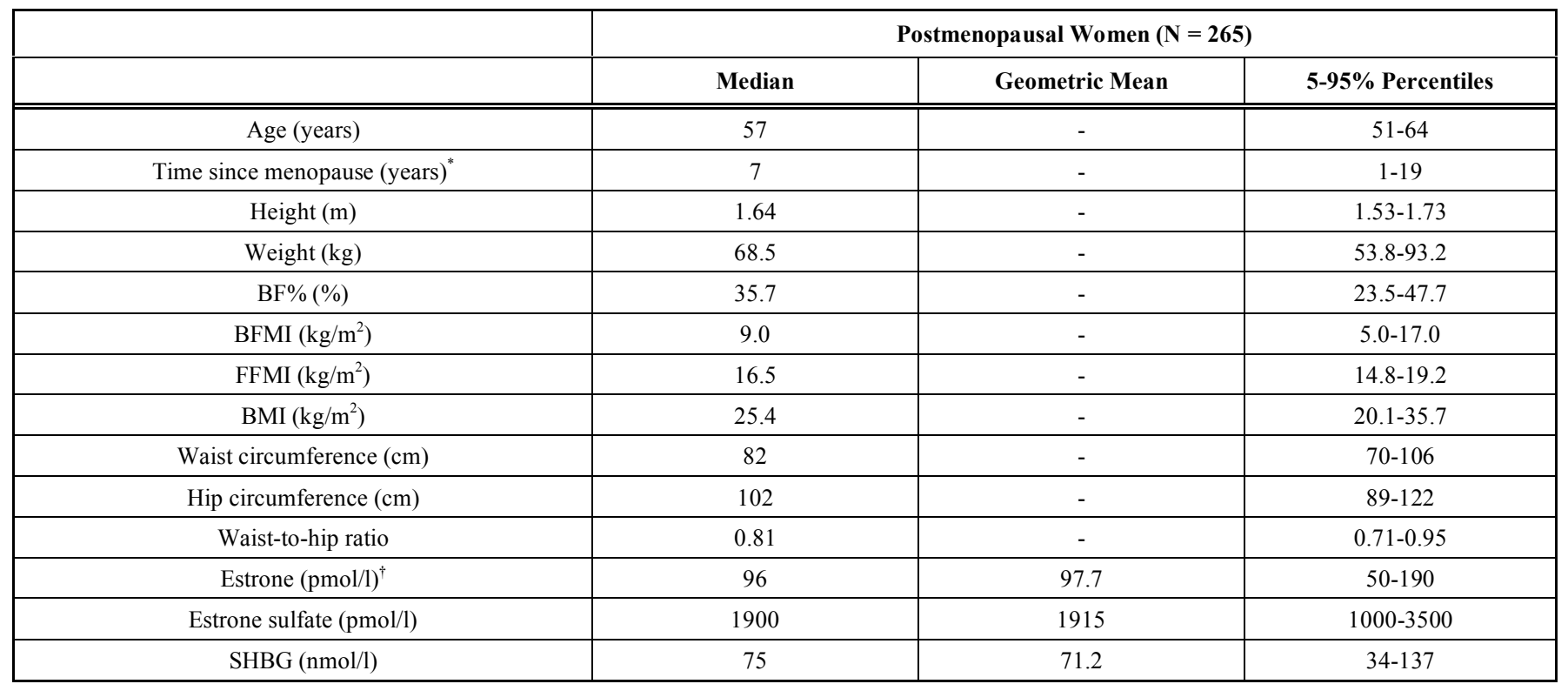

Abbreviations: BFMI, body fat mass index; FFMI, fat free mass index; BF, body fat.

*Time since menopause for 262 women.

${ }^{\dagger}$ Estrone measurements for 263 women. 
missing information on one or more anthropometric measurements and $8(3 \%)$ with missing information for one or more of the potential confounders. Thus, 265 women remained in the study. Two subjects $(1 \%)$ were excluded from the estrone analyses, because they had values under the detection limit.

\section{RESULTS}

Characteristics of the 265 postmenopausal women included in the study are shown in Table 1. The median age at the measurements was 57 years, and the median value for BMI was $25 \mathrm{~kg} / \mathrm{m}^{2}$.

In the multivariate analyses with adjustment for potential confounders, BF\% and BFMI were positively associated with estrone and estrone sulfate and negatively associated with SHBG (Table 2). Similar associations were seen for FFMI. After mutual adjustment, the associations for FFMI were weaker and only the negative association between FFMI and SHBG was statistically significant $(\mathrm{p}=0.05)$.
There were significant linear increases for BMI, waist and hip circumference with estrone and estrone sulfate and significant linear decreases with SHBG. The associations seen for waist circumference were largely unchanged after adjustment for hip circumference, whereas the associations for hip circumference were attenuated towards the null after adjustment for waist circumference.

After stratification on time since menopause, significant positive associations were seen between estrone and BMI, $\mathrm{BF} \%$ and BFMI six or more years after menopause but not during the first five years. No clear differences were seen for associations between estrone sulfate and anthropometric measures by these two levels of time after menopause. For SHBG, the negative associations with $\mathrm{BF} \%$ and BFMI were stronger within 0-5 years after menopause compared to $6+$ years, while a negative association with FFMI was only seen $6+$ years after menopause (data not shown).

A regression model including BFMI and FFMI was not more predictive of any of the two analysed sex-steroid hormones or SHBG than a model with BMI (Estrone: $\mathrm{p}=$ 0.59, Estrone-sulfate: $p=0.98$, SHBG: $p=0.90)$.

Table 2. Age Adjusted and Further Adjusted" Estimates ${ }^{\dagger}$ and 95\% Confidence Intervals for the Association Between Anthropometric Measurements and Levels of Estrogens and SHBG Among 265 Postmenopausal Women From the Danish "Diet, Cancer and Health" Study

\begin{tabular}{|c|c|c|c|}
\hline Anthropometric Predictor & $\begin{array}{c}\text { Estrone } \$ \\
\Delta^{\dagger}(95 \% \text { CI })\end{array}$ & $\begin{array}{c}\text { Estronesulfate } \\
\Delta^{\dagger}(95 \% \text { CI })\end{array}$ & $\begin{array}{c}\text { SHBG } \\
\Delta^{\dagger}(\mathbf{9 5 \%} \text { CI })\end{array}$ \\
\hline \multicolumn{4}{|c|}{ BF\%, per $10 \%$} \\
\hline Adj. $^{*}$ & $8(1 ; 15)$ & $9(3 ; 16)$ & $-22(-28 ;-17)$ \\
\hline Adj. ${ }^{*}+$ FFMI & $7(0 ; 15)$ & $7(0 ; 15)$ & $-18(-24 ;-11)$ \\
\hline Age adj. & $1(0 ; 3)$ & $2(0 ; 3)$ & $-5(-6 ;-4)$ \\
\hline Adj. ${ }^{*}$ & $2(0 ; 3)$ & $2(1 ; 3)$ & $-5(-7 ;-4)$ \\
\hline Adj. ${ }^{*}+$ FFMI & $2(0 ; 3)$ & $2(0 ; 3)$ & $-4(-6 ;-2)$ \\
\hline \multicolumn{4}{|c|}{ FFMI, per $1 \mathrm{~kg} / \mathrm{m}^{2}$} \\
\hline \multicolumn{4}{|c|}{ BMI, per $4 \mathrm{~kg} / \mathrm{m}^{2}$} \\
\hline Age adj. & $4(0 ; 9)$ & $5(2 ; 10)$ & $-15(-19 ;-12)$ \\
\hline Adj.* & $5(1 ; 10)$ & $7(3 ; 11)$ & $-16(-20 ;-12)$ \\
\hline \multicolumn{4}{|c|}{ Waist, per $5 \mathrm{~cm}$} \\
\hline Age adj. & $3(0 ; 5)$ & $3(1 ; 5)$ & $-9(-11 ;-7)$ \\
\hline Adj. & $3(1 ; 5)$ & $3(1 ; 5)$ & $-9(-11 ;-7)$ \\
\hline Adj.* + hip & $3(0 ; 6)$ & $3(0 ; 6)$ & $-11(-14 ;-8)$ \\
\hline \multicolumn{4}{|c|}{ Hip, per 5 cm } \\
\hline Age adj. & $2(0 ; 5)$ & $3(1 ; 5)$ & $-7(-10 ;-5)$ \\
\hline
\end{tabular}

Abbreviations: BFMI, body fat mass index; FFMI, fat free mass index; BF, body fat.

* Adjusted for age (linear), smoking (never, past and current) and alcohol intake (per $10 \mathrm{~g}$ ).

${ }^{\dagger} \Delta$ Estimates reported as percentage change in hormonal measurements per defined unit of change in anthropometric measurement.

$\$$ Estrone measurements for 263 women. 


\section{DISCUSSION}

We confirmed previous findings that BMI is positively associated with estrone and estrone sulfate and negatively associated with SHBG among postmenopausal women. Associations were in the same direction for BFMI and FFMI, and use of these more detailed measures did not give a better prediction of the hormone levels than use of BMI alone.

The anthropometric data used in the present study were obtained in a standard way by trained laboratory technicians. Measurements of sex-steroid hormones were performed in a laboratory with a longstanding experience in measuring female hormones for clinical evaluation. We only had one sample for each individual collected at various hours of the day, but in a previous study, estradiol and SHBG did not show diurnal variation; and hours since last meal and years since storage also had no effect on the concentrations in postmenopausal women [18]. The study subjects were participants from a previous breast cancer case-control study nested within the Diet, Cancer and Health cohort, and though they were not randomly selected from the entire cohort, the controls were randomly selected from specific matching strata. Before combining breast cancer cases and controls into one study group, we tested that the relationships between hormones and anthropometric measurements were similar for cases and controls. The finding of higher levels of estrogens and lower levels of SHBG among breast cancer cases compared to controls is in accordance with many previous studies showing that increased endogenous levels of estrogen and decreased levels of SHBG are associated with an increased risk of breast cancer among postmenopausal women $[19,20]$.

Among 755 postmenopausal non-users of HRT from the Melbourne Collaborative Cohort Study [7], fat mass and fat free mass estimated by bioelectrical impedance analysis were positively associated with free estradiol and estrone sulfate and negatively associated with SHBG, while fat mass, but not fat free mass, was positively associated with total estradiol. The associations with estrogens were mainly seen six or more years after menopause. We also found positive associations between fat mass and fat free mass and estrogen levels and negative associations with SHBG, though these associations tended to be weaker than those found in the Australian study, and the pattern by time since menopause could not be confirmed for estrone sulfate. In another study on 51 postmenopausal women from the control segment of the Women's Alcohol Study who were fed a controlled diet, fat $\%$, central fat and peripheral fat measured by dual energy $\mathrm{X}$-ray absorptiometry were positively associated with estrogens and negatively associated with SHBG, however, these measures did not add further information beyond that of BMI alone [21]. No results were presented for fat free mass. It could have been expected that associations for body fat measures would give a better prediction of estrogens, since such measures give a more precise measure of adiposity than BMI. Our previous study, however, indicated a possible association with lean body mass as well as fat mass since we found that increase in lean body mass was associated with increased risk of breast cancer among postmenopausal women [12].

Several studies have reported positive associations between BMI and female hormones and negative associations with SHBG among healthy postmenopausal women not using HRT [2-4,6,7,18,22,23]. Similar relationships have also been found for waist and hip circumference [2,6,22]. Our results are in agreement with these prior studies. Adipose tissue in the abdominal region and the gluteal region may have different biological effects [24], therefore we adjusted associations with waist circumference for hip circumference and vice versa. Only the associations with waist circumference remained after mutual adjustment. In most studies, female hormones comprise estrone and/or estradiol, while free or non-SHBG bound estradiol were considered in a subset of studies [2-7,23]. Some of these have shown stronger associations with free or non-SHBG bound estradiol than with total estradiol $[2,3,7,23]$. Estrone sulfate was measured in some of the studies of which most showed similar associations with BMI as for estrone and/or estradiol $[4,5,7,23]$ and one showed no association [22]. It is noteworthy that the strongest associations seen in our study were those with SHBG, in the light that SHBG may play a key role in relation to breast cancer, since in addition to being a binding protein for estradiol, SHBG also regulates the action of steroid hormones at several levels [25].

\section{CONCLUSION}

In conclusion, we were able to confirm earlier findings of positive associations between BMI and endogenous estrogen levels and a negative association between BMI and SHBG among postmenopausal women. Associations were in the same direction for both the fat and lean components of BMI and inclusion of these two components did not improve the estimation beyond that of BMI.

\section{ACKNOWLEDGEMENTS}

The study was financially supported by a grant from the Danish Cancer Society (grant OP 00027). We thank Katja Boll, Programmer, and Jytte Fogh Larsen, Secretary, for their help in the collection of data.

\section{FINANCIAL SUPPORT}

The work was supported by the Danish Cancer Society (Grant OP 00027).

\section{REFERENCES}

[1] International Agency for Research on Cancer. Weight Control and Physical Activity. Lyon: IARC Press, 2002.

[2] Bezemer ID, Rinaldi S, Dossus L, et al. C-peptide, IGF-I, sexsteroid hormones and adiposity: a cross-sectional study in healthy women within the European Prospective Investigation into Cancer and Nutrition (EPIC). Cancer Causes Control 2005; 16: 561-72.

[3] Lukanova A, Lundin E, Zeleniuch-Jacquotte A, et al. Body mass index, circulating levels of sex-steroid hormones, IGF-I and IGFbinding protein-3: a cross-sectional study in healthy women. Eur J Endocrinol 2004; 150: 161-71

[4] Madigan MP, Troisi R, Potischman N, Dorgan JF, Brinton LA, Hoover RN. Serum hormone levels in relation to reproductive and lifestyle factors in postmenopausal women (United States). Cancer Causes Control 1998; 9: 199-207.

[5] Hankinson SE, Willett WC, Manson JE, et al. Alcohol, height, and adiposity in relation to estrogen and prolactin levels in postmenopausal women. J Natl Cancer Inst 1995; 87: 1297-302. 
[6] McTiernan A, Wu L, Chen C, et al. Relation of BMI and physical activity to sex hormones in postmenopausal women. Obesity 2006 ; 14: 1662-77.

[7] Baglietto L, English DR, Hopper JL, et al. Circulating steroid hormone concentrations in postmenopausal women in relation to body size and composition. Breast Cancer Res Treat 2009; 115: 171-9.

[8] Key TJ, Appleby PN, Reeves GK, et al. Body mass index, serum sex hormones, and breast cancer risk in postmenopausal women. $\mathrm{J}$ Natl Cancer Inst 2003; 95: 1218-26.

[9] Rinaldi S, KeyTJ, Peeters PH, et al. Anthropometric measures, endogenous sex steroids and breast cancer risk in postmenopausal women: a study within the EPIC cohort. Int J Cancer 2006; 118 : 2832-9.

[10] Tjonneland A and Overvad K. Diet, cancer and health - a population study and establishment of a biological bank in Denmark. Ugeskr Laeger 2000; 162: 350-4.

[11] Nexo BA, Vogel U, Olsen A, et al. A specific haplotype of single nucleotide polymorphisms on chromosome 19q13.2-3 encompassing the gene RAI is indicative of post-menopausal breast cancer before age 55. Carcinogenesis 2003; 24: 899-904.

[12] Mellemkjaer L, Bigaard J, Tjonneland A, et al. Body composition and breast cancer in postmenopausal women: a Danish prospective cohort study. Obesity 2006; 14: 1854-62.

[13] Heitmann B. Prediction of body water and fat in adult Danes from measurement of electrical impedance: a validation study. Int J Obes 1990; 14: 789-802.

[14] VanItallie TB, Yang MU, Heymsfield SB, FunkRC, Boileau RA. Height-normalized indices of the body's fat-free mass and fat mass: potentially useful indicators of nutritional status. Am J Clin Nutr 1990; 52: 953-9

[15] Emment Y, Collins WP, Sommerville JF. Radioimmunoassay of oestrone and oestradiol in human plasma. Acta Endocr 1972; 69: $567-82$.
[16] Franz E, Watson D, Longscope C. Estrone sulphate and dehydroepiandrosterone sulphate concentrations in normal subjects and men with cirrhosis. Steroids 1979; 34: 563-73.

[17] Cornelisse MM, Bennett PE, Christiansen M, et al. Sex hormone binding globulin phenotypes: Their detection and distribution in healthy adults and different conditions. Clin Chim Acta 1994; 225: 115-21.

[18] Verkasalo PK, Thomas HV, Appleby PN, Davey GK, KeyTJ. Circulating levels of sex hormones and their relation to risk factors for breast cancer: a cross-sectional study in 1092 pre- and postmenopausal women (United Kingdom). Cancer Causes Control 2001; 12: 47-59.

[19] Key T, Appleby P, Barnes I, Reeves G. Endogenous sex hormones and breast cancer in postmenopausal women: reanalysis of nine prospective studies. J Natl Cancer Inst 2002; 94: 606-16.

[20] Kaaks R, Rinaldi S, Key TJ, et al. Postmenopausal serum androgens, oestrogens and breast cancer risk: the European prospective investigation into cancer and nutrition. Endocr Relat Cancer 2005; 12: 1071-82.

[21] Mahabir S, Baer DJ, Johnson LL, et al. Usefulness of body mass index as a sufficient adiposity measurement for sex hormone concentration associations in postmenopausal women. Cancer Epidemiol Biomarkers Prev 2006; 15: 2502-7.

[22] Boyapati SM, Shu XO, Gao YT, et al. Correlation of blood sex steroid hormones with body size, body fat distribution, and other known risk factors for breast cancer in post-menopausal Chinese women. Cancer Causes Control 2004; 15: 305-11.

[23] Lamar CA, Dorgan JF, Longcope C, Stanczyk FZ, Falk RT, Stephenson HE, Jr. Serum sex hormones and breast cancer risk factors in postmenopausal women. Cancer Epidemiol Biomarkers Prev 2003; 12: 380-3.

[24] Ballard-Barbash R. Anthropometry and breast cancer. Body size - a moving target. Cancer 1994; 74(Suppl): 1090-100.

[25] Hammond GL. Access of reproductive steroids to target tissues. Obstet Gynecol Clin North Am 2002; 29: 411-23. 